\title{
Near-field wireless sensing of single and multiple open-ended micro coils
}

\author{
A. Yousaf, J. M. Boccard, F. A. Khan, and L. M. Reindl \\ Institute of Microsystems Engineering IMTEK Laboratory for Electrical Instrumentation University of \\ Freiburg, Germany
}

Correspondence to: A. Yousaf (adnan.yousaf@imtek.uni-freiburg.de)

Received: 18 December 2012 - Revised: 21 March 2013 - Accepted: 10 April 2013 - Published: 2 May 2013

\begin{abstract}
In this work we present near-field wireless sensing of single and multiple open-ended micro coils using an electrically small loop antenna. Wirelessly characterized parameters of open-ended micro coils include its resonance frequency, quality factor and inductance. Moreover a wireless frequency-dependent analytical model was developed. Micro coil inductance was extracted from the wirelessly measured signal using a constraint-based least-squares approach. Wireless measurements and analytical fit of micro coils are in strong agreement which validates the analytical model. Finite element method (FEM) simulations of the coupled system were done in COMSOL Multiphysics.
\end{abstract}

\section{Introduction}

Due to device miniaturization the process of testing micro coils becomes a challenging task for the manufacturers since making proper ohmic contact not only requires more concentration for the operating person but also in certain cases deforms the micro coil structure. This wire-based measurement is performed using a standard LCR meter or an impedance analyzer as shown in Fig. 1.

Further, contacting probes also influence the micro coil parameters, which become difficult to eliminate from the actual parameters. This leads to the requirement of investigating new wireless test methods which can be integrated in the manufacturing industry. Several passive read-out systems using inductive and capacitive coupling approaches have been reported frequently. Faul et al. (2011) reported a wireless passive pressure sensor for measuring intraocular pressure where the sensor device consists of a capacitor array and a coil inductor, which form a series LC type resonator. The concept of inductively coupled wireless LC sensors where the stimulus or the applied pressure, strain or torque signal changes the capacitance of an LC circuit, hence shifting the resonance frequency, was reported by Cao et al. (2011); Radislav A Potyrailo and Morris (2009); RamRakhyani et al. (2011); and Ee Lim Tan and Ong (2007).

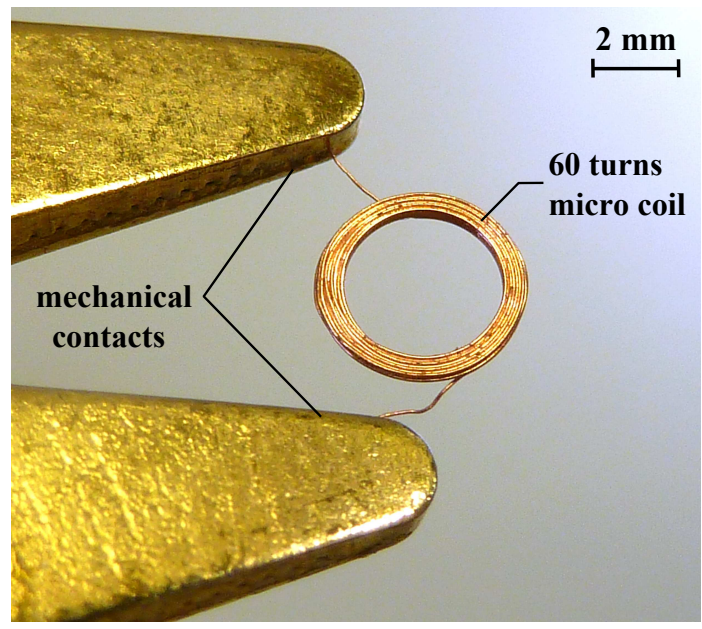

Figure 1. Ohmic contact based wired measurement of a micro coil.

We present here an innovative approach to wirelessly characterize single- and multiple-micro-coil parameters using an electrically small magnetic loop antenna. The remainder of the paper is organized as follows. Section 2 explains the theoretical background of near-field wireless sensing, multilayered micro coils and their applications. In Sect. 3 we discuss the wired characterization of micro coils. Section 4 explains 
the wireless measurement approach and parameter extraction approaches. Measurements and simulation results are discussed in Sect. 5. Conclusion and outlook are discussed in Sect. 6.

\section{Theoretical background}

\subsection{Near-field wireless sensing}

Inductive coupling is one possible approach among several other methods used in near-field wireless sensing applications. In the inductive coupled approach a pick-up sensing coil or an antenna is coupled to the sensing element. In perspective of electromagnetic radiations of an antenna, the space around it can be divided in different regions depending on its electrical size $D$, the operational frequency. For an antenna size larger than the wavelength $(D>\lambda)$, there are three main fields as follows (Balanis, 2007; Davis et al., 2011):

- Reactive near-field region: corresponds to the reactive part of the antenna impedance where the energy is not radiated but stored. The electrical and magnetic fields are out of phase by 180 degrees and non-uniform. The boundary of this field is given by $R=0.62 \sqrt{D^{3} / \lambda}$.

- Radiating near-field (Fresnel) region: the angular field distribution is dependent on the distance. The field decay rapidly as factor of $1 / R^{3}$. As in reactive field, the electric and magnetic fields are out of phase but they start to emerge. The boundary of this field is given $R=2 D^{2} / \lambda$.

- Far-field (Fraunhofer) region: the fields propagate as an electromagnetic wave and are perpendicular to each other and to the direction of propagation as described by the Maxwell equations. The fields decay by a factor of $1 / R$ and the angular distribution does not depend on the distance from the antenna anymore.

However, the regions are different for small antennas $(D<$ $\lambda)$. The radiating near field is inexistent and the boundary between reactive near and far field is given by $R=\lambda / 2 \pi$. Figure 2 shows the simplified field regions for the electrically small single loop antenna used in this work. At $100 \mathrm{MHz}$, the far field would start at $47 \mathrm{~cm}$.

\subsection{Micro coils}

Micro coils are one of the most fundamental passive components used in different electronics devices and applications like transformers, DC-DC converters, optical MEMS, RFID sensors, etc. Further it is one of the effective structures of coil construction in achieving large inductances in small areas. Micro coils can be manufactured using standard micro machining techniques on silicon substrates as discussed in Querol (2012). Several new applications in nearelectromagnetic wireless sensing using micro coils are being

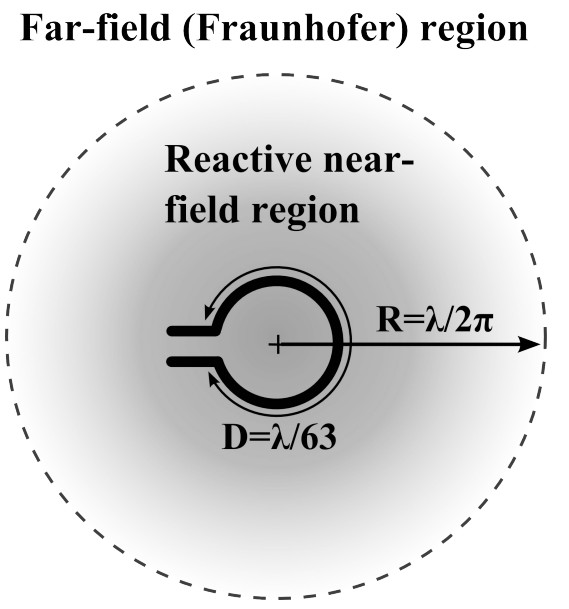

Figure 2. Antenna near- and far-field regions.

reported; one of them is an IOP sensor where a micro coil is implanted in a chip and eye pressure is sensed using a pickup micro coil.

Inductance increase in micro coils can also be enhanced by winding them in multilayers. Micro coil in multilayers are constructed by piling layers of single-layer coils and connecting them. Insulation between these layers is done by using additional layers of insulating materials like polyamide, litz, and polyuntherane by an electroplating process.

Besides insulating the coil wire, it is also coated with a thin layer of magnetic materials like copper or iron. Such a wire is called magnetoplated wire (MPW) and has the following advantages over copper wire without magnetic film plating as discussed in Shinagawa et al. (2009) and Mizuno et al. (2007):

- Increased inductance.

- Higher quality factor.

- Enhanced range of magnetic flux and its linkage.

- Suppression of increase in AC resistance of the coil.

In the presented work the micro coils wirelessly characterized were multilayer air core micro coils having MPW wire insulated with polyutherane and polyamide. Table 1 contains geometrical data of these micro coils, where $h, D_{\mathrm{o}}$ and $W_{\mathrm{d}}$ represents the height, outer diameter and wire diameter of the coil, respectively.

Figure 3 shows the microscopic cross section view of a micro coil with 7 layers.

\section{Wired characterization of micro coils}

Micro coils have several parasitic results due to their structural complexities and the core materials properties. Due to these limitations micro coils require an equivalent circuit 
Table 1. Micro coil geometrical data, Fa. Hopt GmbH.

\begin{tabular}{lrrrrr}
\hline Coil & Turns & Layers & $h[\mathrm{~mm}]$ & $D_{0}[\mathrm{~mm}]$ & $W_{\mathrm{d}}[\mu \mathrm{m}]$ \\
\hline 1 & 33 & 4 & 0.43 & 5.5 & 71 \\
2 & 60 & 8 & 0.6 & 1.95 & 50 \\
3 & 60 & 6 & 0.5 & 4.2 & 50 \\
4 & 100 & 7 & 0.5 & 3.6 & 50 \\
5 & 200 & 10 & 1.18 & 7.5 & 71 \\
6 & 300 & 11 & 1 & 4.6 & 50 \\
7 & 500 & 15 & 2 & 8.2 & 50 \\
\hline
\end{tabular}

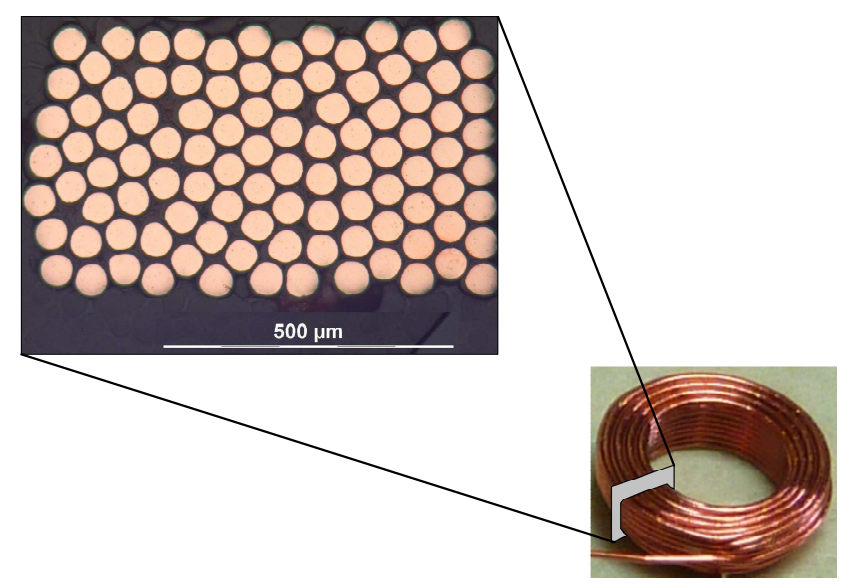

Figure 3. Cross section microscopic view of a micro coil with 7 layers.

model which includes theses parasitic effects. A simplified non-ideal model for small and SMD coils used in different practical applications is shown in Fig. 4.

$R_{\text {ac }}$ represents ac resistance of the coil which is composed of three components, namely dc resistance $R_{\mathrm{dc}}$, winding resistance and skin effects represented as $R_{\mathrm{S}}$ and proximity effect resistance $R_{\mathrm{p}}$ expressed below as

$R_{\mathrm{ac}}=R_{\mathrm{dc}}+R_{\mathrm{s}}+R_{\mathrm{s}} \Omega$.

A multi-turn coil also includes the effects of distributed capacitances. The potential difference that exists between coil winding introduces an electrostatic field distribution between the air and the dielectric material of coil. This effect is approximated equivalently as a capacitor connected across the turns. This capacitance realized at the terminals of an inductor is collectively called the distributed capacitance of the coil. This distributed capacitance introduces dielectric losses which can be modeled by adding a frequency-dependent series resistance to the inductor as discussed in Shishan et al. (2010) and Grandi et al. (1996).

The frequency-dependent complex impedance $Z(w)$ of this model is given below:

$Z(w)=\frac{R_{\mathrm{ac}}+j w L}{1-w^{2} L C_{\mathrm{d}}+j w R_{\mathrm{ac}} C_{\mathrm{d}}}$

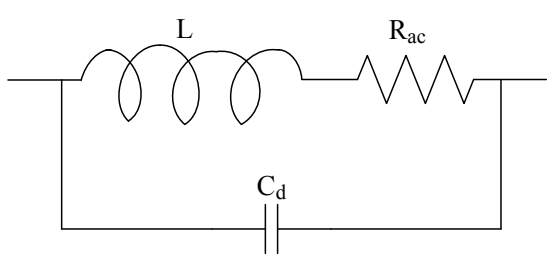

Figure 4. Simplified non-ideal micro coil electrical model (Grandi et al., 1996; Naishadham, 2011).

with the angular frequency $w=2 \pi f$.

The impedance model (2) is split in real $Z_{r}$ and imaginary $\mathrm{Z}_{i}$ parts as follows:

$\operatorname{Re}(Z)=\frac{R_{\mathrm{ac}}}{\left(1-w^{2} L C_{\mathrm{d}}\right)^{2}+w^{2}\left(C_{\mathrm{d}} R_{\mathrm{ac}}\right)^{2}}$

$\operatorname{Im}(Z)=\frac{w L\left(1-w^{2} L C_{\mathrm{d}}-\frac{C_{\mathrm{d}} R_{\mathrm{ac}}^{2}}{L}\right)}{\left(1-w^{2} L C_{\mathrm{d}}\right)^{2}+w^{2}\left(C_{\mathrm{d}} R_{\mathrm{ac}}\right)^{2}}$.

The distributed capacitance $C_{\mathrm{d}}$ causes the inductor to resonate at $W_{\text {res }}$ with a quality factor $Q_{0}$. Both of these parameters can be explicitly obtained from the measured impedance signal.

Resonance frequency and quality factor of the model can be obtained as follows:

$w_{\text {res }}=\sqrt{\frac{1}{L C_{\mathrm{d}}}-\frac{R_{\mathrm{ac}}^{2}}{L^{2}}}$

$Q_{0}=\frac{w_{\text {res }}}{\Delta w}$.

The $Q$ factor is computed from the measured resonance frequency and $3 \mathrm{~dB}$ half-power bandwidth.

For the case when the $R_{\mathrm{ac}}^{2} / L^{2} \ll 1 / L C_{\mathrm{d}}$, the resonance frequency expression is the same as for a series RLC network. The resonance frequency $W_{\text {res }}$ in Eq. (5) can be expressed in terms of quality factor as follows:

$W_{\text {res }}=\frac{1}{\sqrt{L_{0} C_{\mathrm{d}}}} \cdot \sqrt{\frac{Q^{2}}{1+Q^{2}}}$.

The impedance model given in Eq. (2) can also be expressed in terms of resonance frequency as follows:

$Z(w)=\frac{R_{\mathrm{ac}}+j w L^{2} C_{\mathrm{d}}\left(w_{\mathrm{res}}^{2}-w^{2}\right)}{\left(1-w^{2} L C_{\mathrm{d}}\right)^{2}+w^{2}\left(C_{\mathrm{d}} R_{\mathrm{ac}}\right)^{2}}$.

At the resonance frequency $w=w_{\text {res }}$, inductance $L_{0}$ and resistance $R_{0}$ can be obtained as follows:

$R_{0}=\frac{Z_{\text {res }}}{1+Q^{2}}$ 
Table 2. Computed and measured parameters using resonance frequency definitions Case 1 and 2 for a micro coil with 33 turns.

\begin{tabular}{lrrr}
\hline Parameter & Case 1 & Case 2 & \% Difference \\
\hline$f_{\text {res }}[\mathrm{MHz}]$ & 50.982 & 50.981 & 0.002 \\
$Z_{\text {res }}[\mathrm{k} \Omega]$ & 37.11 & 37.62 & 1.35 \\
$Q$ factor & 13.75 & 13.79 & 0.29 \\
$R_{0}[\Omega]$ & 195.25 & 197 & 0.88 \\
$L_{0}[\mu \mathrm{H}]$ & 8.67 & 8.76 & 1 \\
$C_{\mathrm{d}}[\mathrm{pF}]$ & 1.19 & 1.181 & 0.75 \\
\hline
\end{tabular}

$L_{0}=\frac{Q R_{0}}{w_{\text {res }}}$.

The parallel distributed parasitic capacitance $C_{\mathrm{d}}$ that models the turn-to-turn and turn-to-core capacitance is treated as frequency invariant. Regarding the first dominant resonance frequency, capacitance can be explicitly obtained from measured data as given below (Naishadham, 2011):

$$
C_{\mathrm{d}} \triangleq C\left(w_{\mathrm{res}}\right)=\frac{1}{w_{\mathrm{res}}^{2} L_{0}} \cdot \frac{Q^{2}}{1+Q^{2}} .
$$

The resonance frequency can be determined from the measured data using the following two basic definitions:

- Case 1: frequency at which impedance imaginary part $\operatorname{Im}(Z) \approx 0$.

- Case 2: frequency at which impedance real part $\operatorname{Re}(Z)$ is maximum.

In practice it is easier to measure the peak or maximum of impedance real part rather than measuring the zero of $\operatorname{Im}(Z)$ as discussed in Nopper et al. (2011). Equations (7)-(11) are used to evaluate the model parameters at the measured resonance frequencies and impedances. Table 2 contains the measured and computed parameters for both definitions of resonance frequency.

As evident from data in Table 2, the discrepancy in resonance frequencies for both cases is $0.002 \%$ for a 33-turn micro coil having a $Q$ factor of 13.95 . This discrepancy increases at maximum to $0.98 \%$ for a micro coil with 500 turns having $Q$ factor of 4.75 . This increase of discrepancy in resonance frequency is not a measurement error but due to the low $Q$ factor of the micro coil. Figure 5 shows the relative error $\Delta Q / Q$ plotted against the measured $Q$ factor values.

Wired-measurement and calculated parameters of different micro coils are given in Table 3. Figures 6 and 7 show the wired impedance (real and imaginary) measurement and parametric fit.

\section{Wireless measurement approach}

A single-turn electrically small circular loop antenna was used to inductively couple the open-ended micro coils as dis-

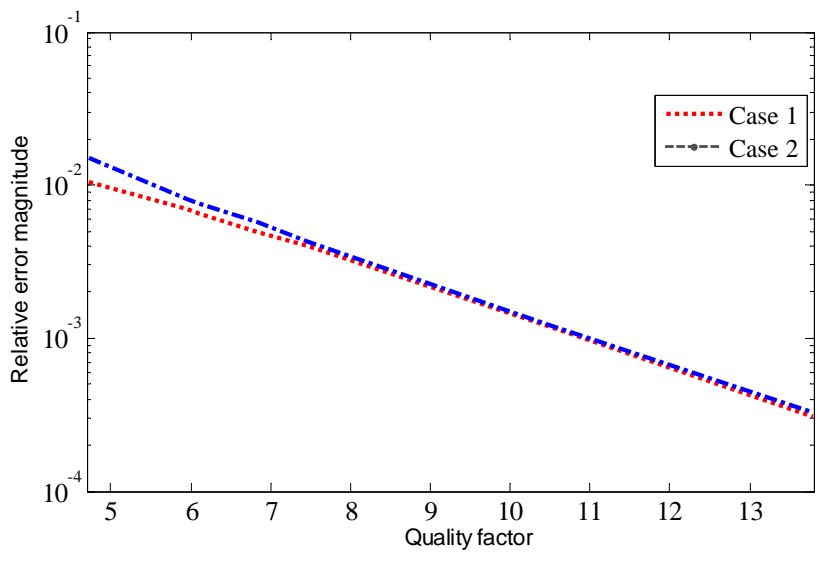

Figure 5. Relative Quality factor error $\Delta Q / Q$ for both cases of resonance frequency. Case 1: $\operatorname{Re}(Z)$ maximum; Case 2: $\operatorname{Im}(Z)$ minimum.

Table 3. Measured and computed parameters of different multilayer micro coils.

\begin{tabular}{lrrrrr}
\hline Coil & $f_{\text {res }}[\mathrm{MHz}]$ & $Q$ & $L_{0}[\mu \mathrm{H}]$ & $C_{0}[\mathrm{pf}]$ & $R_{0}[\mathrm{k} \Omega]$ \\
\hline 1 & 50.98 & 13.79 & 8.76 & 1.11 & 0.197 \\
2 & 64.73 & 7.47 & 5.91 & 1.01 & 0.322 \\
3 & 34.53 & 10.46 & 26.9 & 0.8 & 0.558 \\
4 & 43.69 & 6.1 & 28.71 & 0.45 & 1.29 \\
5 & 6.44 & 4.73 & 270 & 2.16 & 2.314 \\
6 & 8.17 & 5.96 & 390 & 0.97 & 3.367 \\
7 & 3.351 & 6.83 & 1451 & 1.52 & 4.47 \\
\hline
\end{tabular}

cussed in Yousaf and Reindl (2011). Figure 8 shows pictorially the measurement overview for near-field coupled openended micro coils. The wirelessly measured impedance signal for the open-ended coil under test includes the effects of coupling capacitance between the loop antenna and micro coil. The signal measurement is performed using a network analyzer E5071B (Agilent, 2007). The resonance frequency and quality factor of the micro coil were obtained from the wirelessly measured reflected impedance signal. Inductance and loss resistance were extracted from the measured impedance signal. Wired micro coil measurements as discussed in Sect. 3 provide a reference to compute the tolerance range between the wirelessly and wired measurements of micro coils. Micro coils' parameters' tolerance range $T_{\text {tol }}$ can be computed as follows:

$T_{\text {tol }}=\frac{P_{\text {wired }}-P_{\text {wireless }}}{P_{\text {wired }}} \%$

\subsection{Wireless analytical model}

The equivalent electrical circuit model of the inductively coupled micro coil with the loop antenna is shown in Fig. 9. The open-ended micro coil is modeled as a series RLC network coupled to the loop antenna. $L_{2}$ and $R_{2}$ are the 


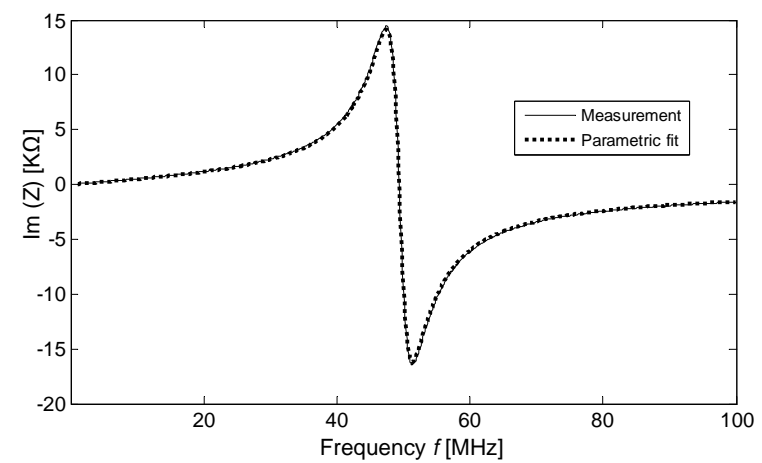

Figure 6. Measured impedance imaginary part and analytical fit for a micro coil with 33 turns and resonance frequency of $50.98 \mathrm{MHz}$.

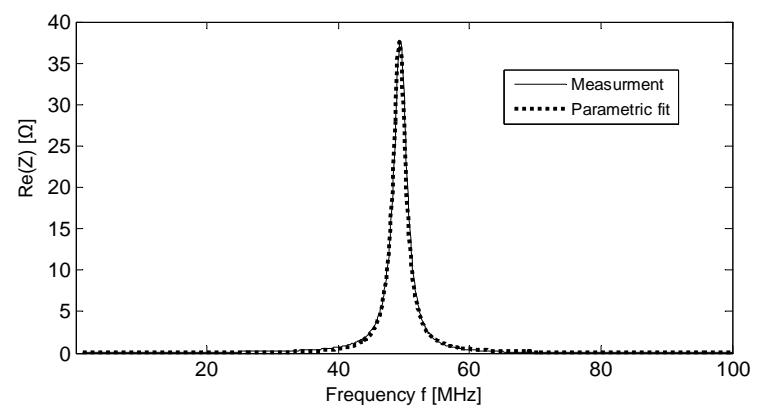

Figure 7. Measured impedance real part and analytical fit for a micro coil with 33 turns and resonance frequency of $50.98 \mathrm{MHz}$.

inductance and loss resistance of the micro coil, where $C_{x}$ is the capacitance of the micro coil including the coupling capacitance (gimmick effect) between the micro coil and loop antenna conductor. Further, $R_{1}$ and $L_{1}$ represent the resistance and inductance of the loop antenna. In this model the loop antenna capacitance is ignored as the wireless measurements performed to test micro coils is well below selfresonating frequency of loop antenna, i.e.1.59 GHz.

Wireless analytical model of the coupled system is derived by applying KVL law and standard transformer equations. The complex impedance $Z_{1}=U_{1} / I_{1}$ of the coupled system including the effects of reflected impedance can be represented in terms of wirelessly measured resonance frequency $W_{\text {res }}$ and quality factor as

$Z_{1}=R_{1}+j w L_{1}\left(1+\frac{\left(\frac{w}{w_{\text {res }}}\right)^{2}(k n)^{2}}{1-\left(\frac{w}{w_{\text {res }}}\right)^{2}+\frac{j}{Q_{2}} \frac{w}{w_{\text {res }}}}\right)$,

where $k$ is the electromagnetic coupling coefficient given in Eq. (14):

$k=\frac{M}{\sqrt{L_{1} L_{2}}}$.

$M$ is the mutual inductance between the coupled coils, where $n$ is the transformer turns ratio $n=N_{\mathrm{s}} / N_{\mathrm{s}}$ between the excitation and micro coil.

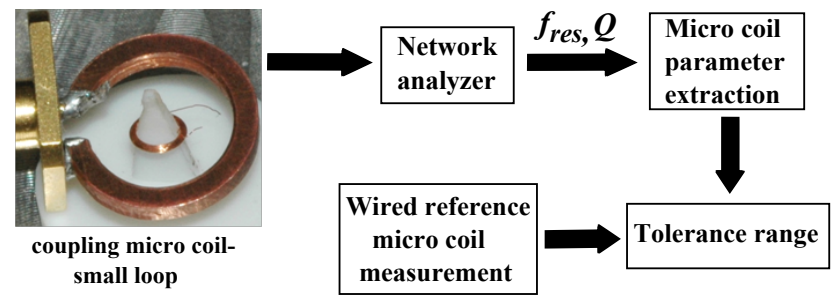

Figure 8. Wireless measurement approach overview.

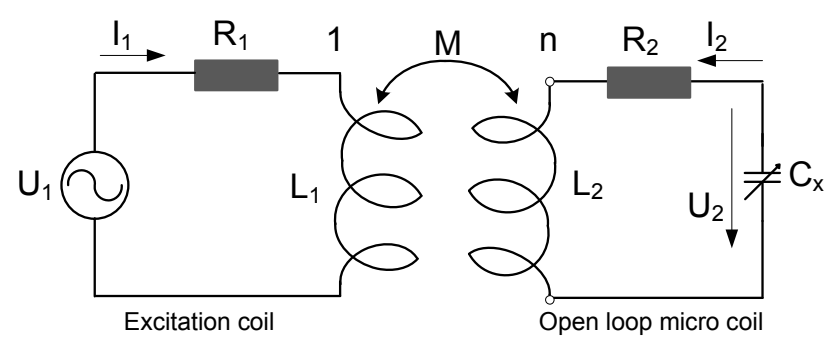

Figure 9. Equivalent electrical circuit model of the inductively coupled single micro coil with an electrically small loop antenna.

Analytical evaluation of micro coil resonance frequency from the wirelessly measured impedance imaginary part is difficult due to its increased complexity. Therefore the real part of the impedance is used (given in Eq. 15), which provides a fair analytical estimation for micro coil resonance frequency:

$$
\operatorname{Re}\left\{Z_{1}\right\}=R_{1}+\frac{L_{1}(k n)^{2} w^{4}}{Q_{2}\left[\left(1-\frac{w^{2}}{w_{\mathrm{res}}^{2}}\right)^{2}+\left(\frac{w}{Q_{2} w_{\mathrm{res}}}\right)^{2}\right] w_{\mathrm{res}}^{3}} .
$$

Analytically the resonance frequency of the micro coil $W_{\text {res }}$ can be obtained by taking the maximum of Eq. (15), $\operatorname{Re}\left\{Z_{1}\right\}_{\max \rightarrow w}$, with respect to frequency as

$W_{\max }=\frac{2 Q_{2}}{\sqrt{4 Q_{2}^{2}-1}} w_{\text {res }}$.

For the values of $Q_{2}$ above 3 the maximum of the real part of impedance is approximately equal to the resonance frequency. The error in resonance frequency reduces to less than $1 \%$ for the micro coils having a $Q$ factor greater than 3 .

The quality factor of the open-ended micro coils can also be approximated from the real part of impedance by using half-power band width approximation as given in Eq. (6).

\subsection{Parameter estimation}

Micro coil resonance frequency $W_{\text {res }}$ and quality factor $Q_{2}$ are directly measured from the wireless impedance measurement as discussed previously. However, its remaining parameters, i.e. inductance, resistive loss and capacitance, cannot be directly measured. 


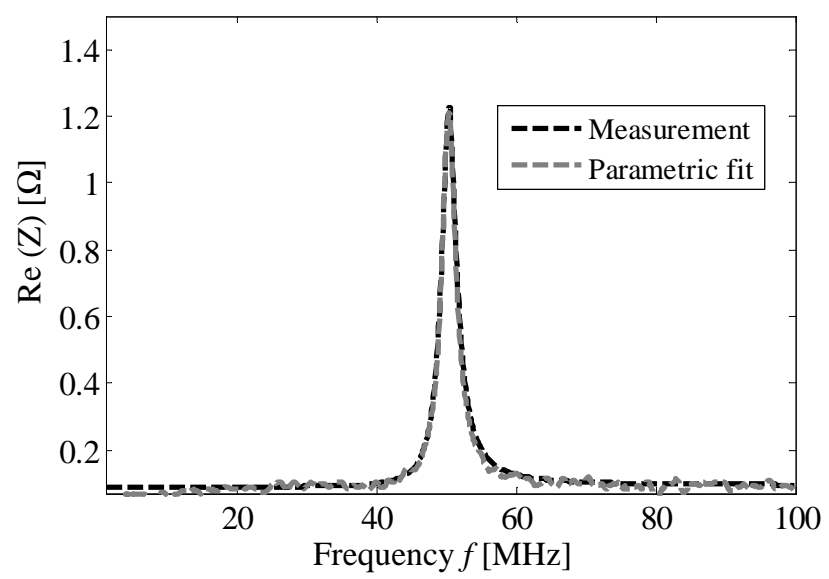

Figure 10. Wirelessly measured impedance real part and analytical fit for a micro coil with resonance frequency of $51.19 \mathrm{MHz}$ with an error of $0.41 \%$.

A least-squares parameter estimation approach is used to extract the unknown parametric values of micro coils (Yousaf, 2012). This estimation routine locates the global minimum of the function expressed as the sum of least sqaures. The impedance model in Eq. (13) is modified to the impedance extraction model $Z_{\text {ext }}$ as follows:

$Z_{\mathrm{ext}}=R_{1}+j w L_{1}\left(1+\frac{\left(\frac{w}{w_{\mathrm{res}}}\right)^{2}(k n)^{2}}{1-\left(\frac{w}{w_{\mathrm{res}}}\right)^{2}+\frac{j}{Q_{2}^{2}} \frac{L_{2}}{R_{2}}}\right)$.

The impedance extraction model allows optimally approximating the data model with the measured signal. The modified form given in Eq. (17) introduces inductance $L_{2}$ and resistance $R_{2}$ as extraction parameters holding the following relationship:

$\frac{j}{Q_{2}} \cdot \frac{w}{w_{\text {res }}} \approx \frac{j}{Q_{2}^{2}} \cdot \frac{L_{2}}{R_{2}}$.

The frequency-dependent capacitance $C_{x}$ is not introduced in the extraction model, hence reducing the degree of freedom to two instead of three. The capacitance $C_{x}$ can be analytically calculated using the measured resonance and extracted inductance as follows:

$C_{x} \approx w_{\text {res }}^{2} \cdot L_{2}$.

Figures 10 and 11 show the parametric model (real and imaginary) and wireless measurement of a micro coil having a resonance frequency of $51.19 \mathrm{MHz}$ with an error of $0.41 \%$ from the actual resonance frequency of the micro coil.

\subsection{Wireless measurement of multiple open-ended micro coil}

Simultaneously measuring more than one micro coil provides an additional measurement possibility to test the same

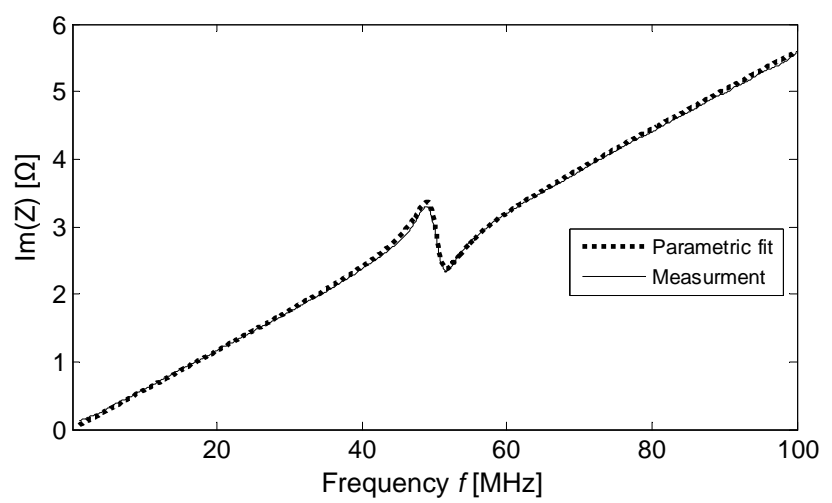

Figure 11. Wirelessly measured impedance imaginary part and analytical fit for a micro coil with resonance frequency of $51.19 \mathrm{MHz}$ with an error of $0.41 \%$.

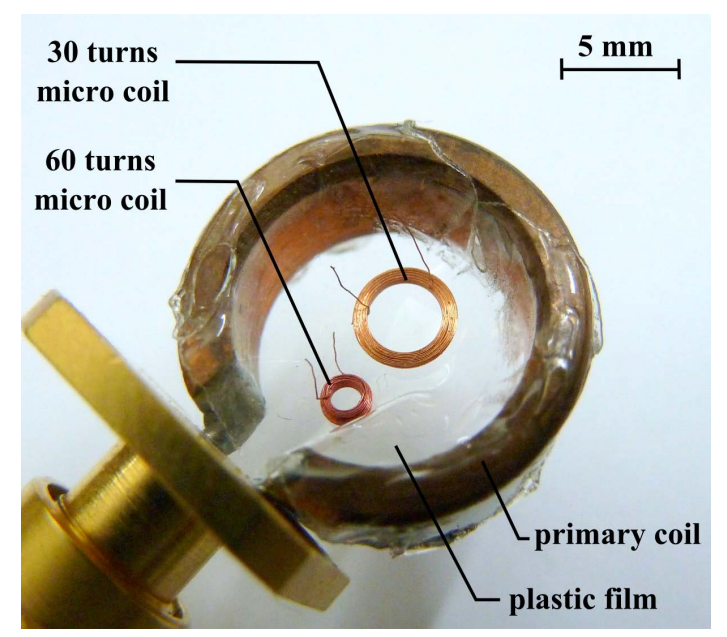

Figure 12. Simultaneous wireless measurement of two different open-ended micro coils placed inside electrically small loop antenna.

and different types of open-ended micro coils. Figure 12 shows 2 different dimensions of open-ended micro coils placed inside the loop antenna for simultaneous measurement of their resonance frequencies and $Q$ factors.

Figure 13 shows the measured resonance frequencies of two different coils having a frequency separation of 13.82 MHz. In multiple-micro-coil simultaneous measurements the capacitive coupling between the multiple micro coils and loop antenna is enhanced, due to which the resonance frequencies of the micro coils is shifted from their actual values. Figure 14 shows the comparison between a measurement of single open-ended micro coil and the simultaneous measurement of multiple open-ended micro coil. For the same micro coils' simultaneous measurement with a loop antenna, the resonance frequency shifts from $50.19 \mathrm{MHz}$ to $49.93 \mathrm{MHz}$, having an increased deviation $\sigma$ of $1.8 \%$ from the actual resonance frequency. 


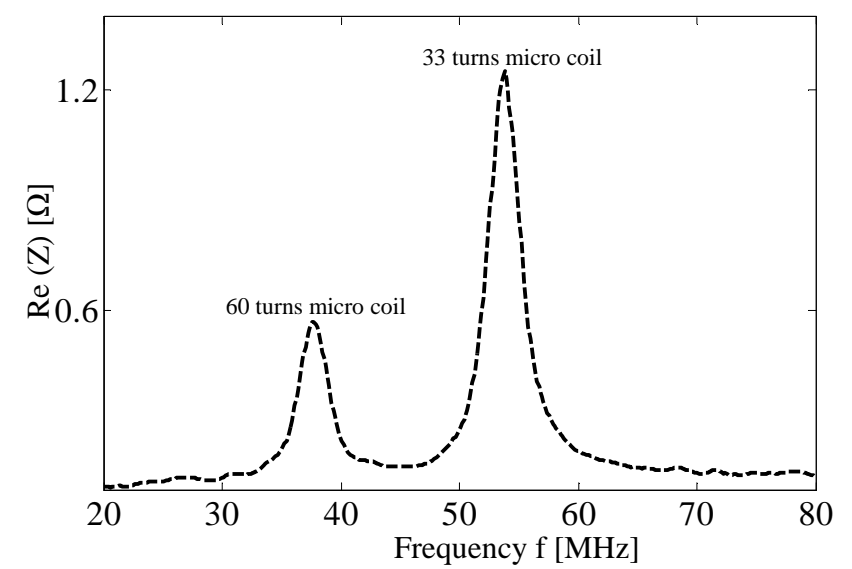

Figure 13. Simultaneously measured wireless resonance frequencies of two different micro coils with 33 and 60 turns using electrically small loop antenna.

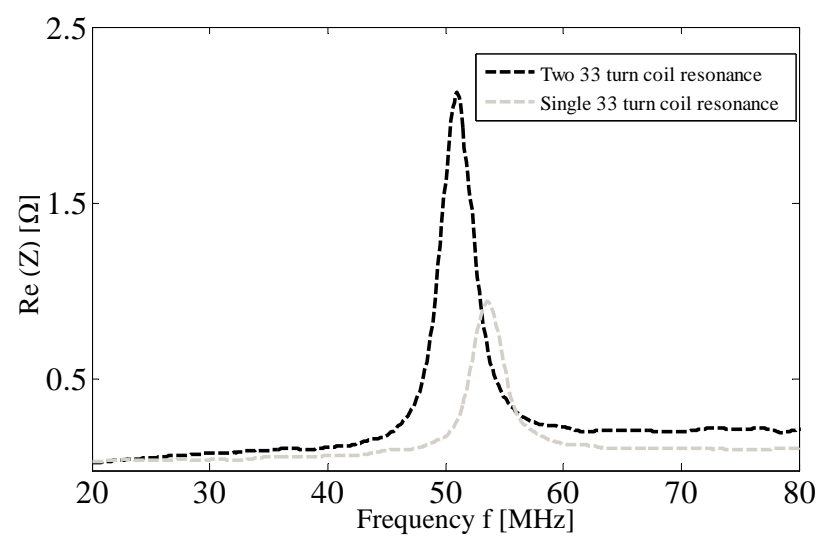

Figure 14. Two 33-turn open-loop micro coil with measured resonance frequency of 49.93 [MHz] plotted vs single 33-turn micro coil with measured resonance frequency of 51.97 [MHz].

\section{Measurements and simulations}

Micro coils are wirelessly measured in a frequency range from 1 to $100 \mathrm{MHz}$. Wirelessly measured, extracted and calculated parameters of the test micro coils characterized using an electrically small loop antenna are given in Table 4 .

Random errors in resonance frequencies of multiplemicro-coil simultaneous measurement increase compared to the single-micro-coil measurement. Figures 15 and 16 depict the random errors calculated for a 33-turn singlemicro-coil measurement and multiple-micro-coil simultaneous measurements.

To simulate the presented problem of measurement approaches based on open-ended single and multiple micro coils, a COMSOL 2-D asymmetrical approach is used. A small loop antenna is modeled by using a copper square 2-D surface. The open-ended condition of the micro coil is approximated by applying zero magnetic field conditions on the
Table 4. Wirelessly characterized parameters of open-ended micro coils inductively coupled to an electrically small loop antenna.

\begin{tabular}{lrrrrr}
\hline Coil & $f_{\text {res }}[\mathrm{MHz}]$ & $Q$ & $L_{2}[\mu \mathrm{H}]$ & $C_{x}[\mathrm{pf}]$ & $R_{2}[\Omega]$ \\
\hline 1 & 51.25 & 13.42 & 8.54 & 1.13 & 181 \\
2 & 65.73 & 7.13 & 5.31 & 1.095 & 283 \\
3 & 36.13 & 10.16 & 25.3 & 1.2 & 458 \\
4 & 44.19 & 5.82 & 26.12 & 0.32 & 1012 \\
5 & 6.82 & 4.44 & 263 & 2.32 & 2218 \\
6 & 8.32 & 5.42 & 379 & 0.92 & 3196 \\
7 & 3.82 & 6.83 & 1382 & 1.32 & 4420 \\
\hline
\end{tabular}

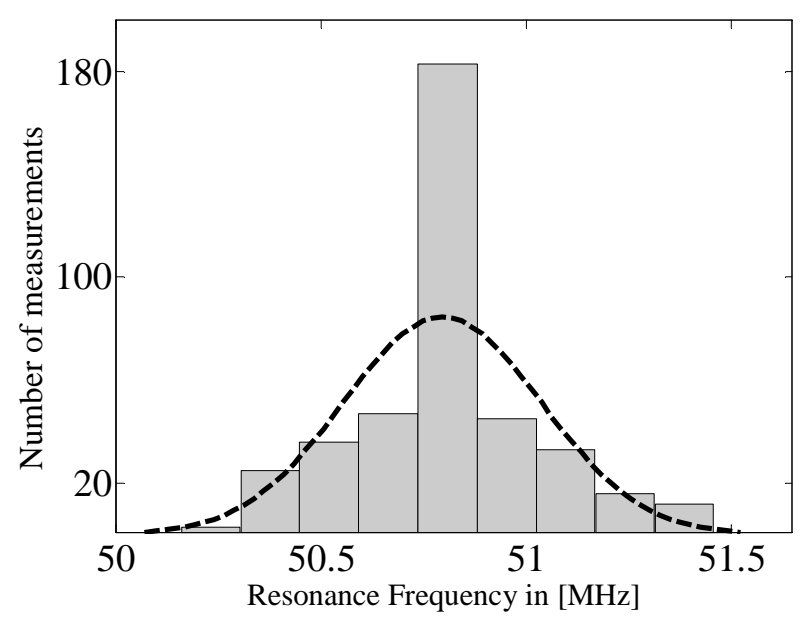

Figure 15. Error distributions in wirelessly measured resonance frequency of a single micro coil with 33 turns measured at the center of loop antenna with mean resonance frequency of $50.82 \mathrm{MHz}$. The dotted line represents the Gaussian distribution of the corresponding moments.

2-D array of the micro coil (Trezise, 2011; Graf et al., 2009). An AC excitation signal of $1 \mathrm{dBm}$ is applied to the loop antenna, hence generating the magnetic flux $\Phi$ which is linked to the micro coils. Magnetic field behavior and the magnetic flux linkage of the inductively coupled test micro coils with the loop antenna is shown in Fig. 17. The arrows and the stream lines show the magnetic flux and linkage through the air. The magnetic flux $\Phi_{\text {testcoil }}$ and current $I$ passing through the micro coils are calculated at the constructed 2-D coil array boundary. Individual micro coil inductances $L_{\text {sim }}$ can be calculated as follows (Ghali and Rehman, 2009):

$L_{\text {sim }}=\frac{\Phi_{\text {testcoil }}}{I} \times N^{2}$,

where $N$ is the micro coil number of turns.

The multilayered micro coils' turns are close together as shown in the cross section microscopic view (Fig. 3); this maintains a high level of flux linkages between the turns. This implies a general rule that the inductance increases with the square of the number of turns (Bowtell and Robyr, 1998). 


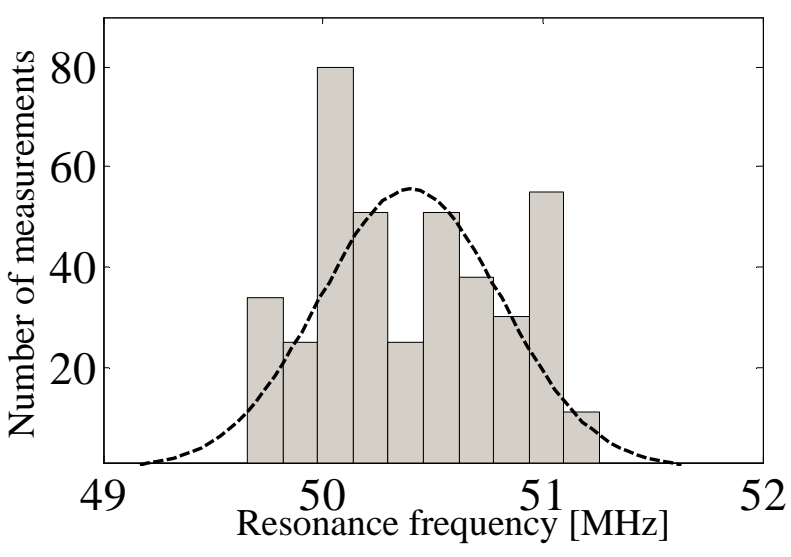

Figure 16. Error distributions in wirelessly measured resonance frequency of a micro coil with 33 turns measured at the center of loop antenna in presence of another test micro coil with 60 turns. The mean resonance frequency of the 33-turn micro coil is shifted to $50.12 \mathrm{MHz}$. The dotted line represents the Gaussian distribution of the corresponding moments.

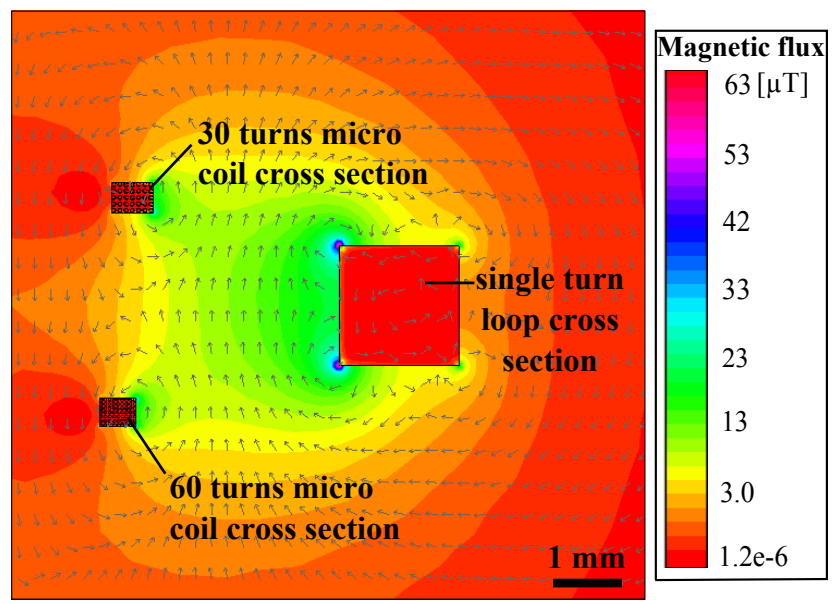

Frequency: $52 \mathrm{MHz}$ Input power: $1 \mathrm{dBm}$

Figure 17. 2-D FEM simulation of 60- and 30-turn micro coils coupled to a small loop antenna.

Figure 18 shows the comparison of wirelessly measured and simulated inductances of micro coils.

\section{Conclusions}

We report here a wireless characterization technique of openended single and multiple micro coils, using an electrically small loop antenna. A wireless analytical model is developed for the coupled system and compared with the measurements, which are in strong agreement, hence validating the model. Detection of multiple resonance frequencies for simultaneous measurement of open-ended micro coils is also presented here. The multiple-micro-coil simultaneous mea-

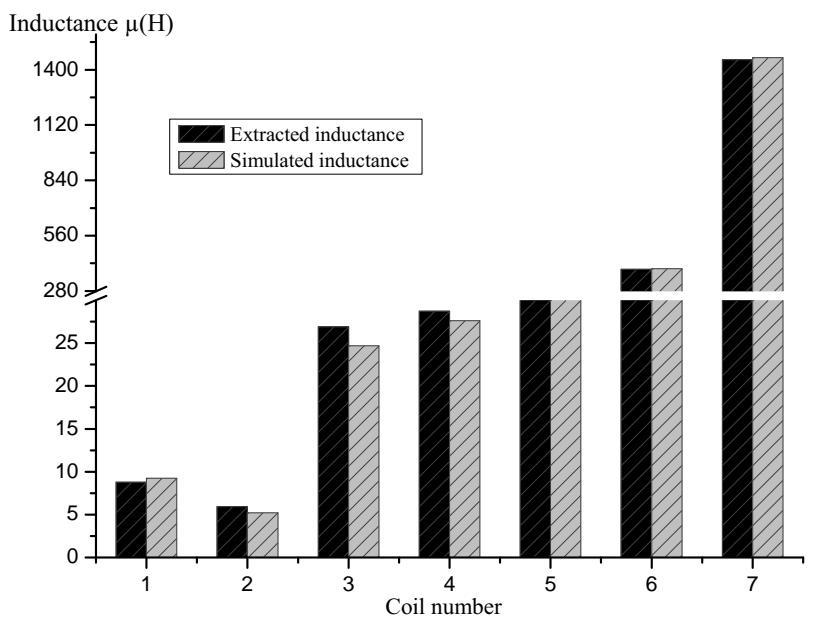

Figure 18. Wirelessly extracted and numerically computed inductance values of different open-ended micro coils.

surement has high coupling capacitance with increased relative errors in resonance frequencies of test micro coils.

Relative errors in resonance frequency, and $Q$ factor of the micro coils ranged from $1.2 \%$ to $4.1 \%$ and $0.92 \%$ to $3.2 \%$, respectively, for the single- and multiple-micro-coil measurement approaches.

Acknowledgements. The authors would like to thanks German Federation of Industrial Research Associations AIF for supporting and facilitating the research project.

Edited by: M. Kraft

\section{References}

Ghali, H. A. and Rehman, H. A.: Understanding Mutual Inductance using COMSOL Multiphysics Milan, 2009.

Agilent: Installation and Quick Start Guide, 7th Edition, 2007.

Balanis, C. A.: Antenna Theory Analysis and Design, vol. 3, Wiley Interscience, 2007.

Bowtell, R. and Robyr, P.: Multilayer Gradient Coil Design, Journal of Magnetic Resonance, 131, 286-294, 1998.

Cao, H., Weber, R., and Hamouche, N.: A passive intraocular pressure sensor and a wireless sensing technique using an intermediate LC resonator, in: Life Science Systems and Applications Workshop (LiSSA), 2011 IEEE/NIH, 5-8, doi:10.1109/LISSA.2011.5754141, 2011.

Davis, W., Yang, T., Caswell, E., and Stutzman, W.: Fundamental limits on antenna size: a new limit, Microwaves, Antennas Propagation, IET, 5, 1297-1302, doi:10.1049/iet-map.2010.0604, 2011.

Ee Lim Tan, Wen Ni Ng, R. S. B. D. P., and Ong, K. G.: A Wireless, Passive Sensor for Quantifying Packaged Food Quality, Sensors Journal, 7, 1747-1756, 2007.

Faul, A., Turner, M., and Naber, J.: Implantable wireless microsystems for the measurement of intraocular pressure, in: Circuits and Systems (MWSCAS), 2011 IEEE 54th International Midwest 
Symposium on, 1-4, doi:10.1109/MWSCAS.2011.6026619, 2011.

Graf, T., Schalli, O., Furrer, A., and Marty, P.: Inductance of Magnetic Plated Wires as a Fucntion of Frequency and Plating Thicknes Milan, Proceedings of the COMSOL Conference 2009 Milan, 2009.

Grandi, G., Kazimierczuk, M., Massarini, A., and Reggiani, U.: Stray capacitances of single-layer air-core inductors for highfrequency applications, in: Industry Applications Conference, 1996, Thirty-First IAS Annual Meeting, IAS '96., Conference Record of the 1996 IEEE, vol. 3, 1384-1388, vol. 3, doi:10.1109/IAS.1996.559246, 1996.

Mizuno, T., Enoki, S., Hayashi, T., Asahina, T., and Shinagawa, H.: Extending the Linearity Range of Eddy-Current Displacement Sensor With Magnetoplated Wire, Magnetics, IEEE Transactions on, 43, 543-548, doi:10.1109/TMAG.2006.887853, 2007.

Naishadham, K.: Closed-Form Design Formulas for the Equivalent Circuit Characterization of Ferrite Inductors, Electromagnetic Compatibility, IEEE Transactions on, 53, 923-932, doi:10.1109/TEMC.2011.2116795, 2011.

Nopper, R., Has, R., and Reindl, L.: A Wireless Sensor Readout System 02014;Circuit Concept, Simulation, and Accuracy, Instrumentation and Measurement, IEEE Transactions on, PP, 1-8, doi:10.1109/TIM.2011.2122110, 2011.

Querol, J. M. A.: Dissertation,University Poltecnica de Catalunya BarcelonaTech,Monitoring Switch Type Sensors and Powering Autonomous Sensors via Inductive coupling. Applications to Removable Seats in Vehicles June, 2012.
Radislav, A. Potyrailo, C., and Morris, W. G.: Combinatorial Screening of Polymeric Sensing Materials Using RFID Sensors: Combined Effects of Plasticizers and Temperature, American Chemical Society, 2009.

RamRakhyani, A., Mirabbasi, S., and Chiao, M.: Design and Optimization of Resonance-Based Efficient Wireless Power Delivery Systems for Biomedical Implants, Biomedical Circuits and Systems, IEEE Transactions on, 5, 48-63, doi:10.1109/TBCAS.2010.2072782, 2011.

Shinagawa, H., Suzuki, T., Noda, M., Shimura, Y., Enoki, S., and Mizuno, T.: Theoretical Analysis of AC Resistance in Coil Using Magnetoplated Wire, Magnetics, IEEE Transactions on, 45, 3251-3259, doi:10.1109/TMAG.2009.2021948, 2009.

Shishan, W., Zeyuan, L., and Yan, X.: Extraction of parasitic capacitance for toroidal ferrite core inductor, in: Industrial Electronics and Applications (ICIEA), 2010 the 5th IEEE Conference on, 451-456, doi:10.1109/ICIEA.2010.5517152, 2010.

Trezise, T.: Modelling Inductively Coupled Coils for Wireless Implantable Bio-Sensors, A Novel Approach Using the Finite Element Method, M.Sc Thesis University of Victoria Canada, 2011.

Yousaf, A.: Passive Wireless Sensing of Micro coil parameters in: fluidic environments,Sensors and Actuators A: Physical, Elsevier, doi:10.1016/j.sna.2012.01.032, 2012.

Yousaf, T. J. and Reindl, P. L.: Wireless Measurement System for Extracting Open Loop Micro Coil Parameters,FREQUENZ, Journal of RF/Microwave Engineering, Photonics and Communications, Special Issue GeMiC2011, 2011. 